\title{
Sistematización del Proyecto Intercátedras “Es Asunto de Todos y de Todas". Una forma de promover el respeto a los derechos de la niñez y la adolescencia desde la Escuela de Ciencias Sociales y Humanidades de la UNED
}

\section{Ana Lorena Vargas Cubero ${ }^{1}$ y Gabriela Villalobos Torres ${ }^{2}$}

1. Bachiller en la Enseñanza de los Estudios Sociales y la Cívica. Licenciada en Educación Cívica. Máster en Administración Educativa de la Universidad de Costa Rica. Máster en Evaluación Educativa de la U. Magister. Egresada del Doctorado en Tecnología Instruccional y Educación a Distancia de Nova Southeastern University. Actualmente labora como Directora de la Cátedra de Geografía y Cívica de la Escuela de Ciencias Sociales y Humanidades de la Universidad Estatal a Distancia. Correo electrónico: avargas@uned.ac.cr

2. Bachiller y licenciada en Trabajo Social de la Universidad de Costa Rica. Magister en Violencia Intrafamiliar y de Género de la Universidad de Costa Rica. Actualmente labora como Directora de la Cátedra de Trabajo Social de la Escuela de Ciencias Sociales y Humanidades de la Universidad Estatal a Distancia. Correo electrónico: gvillalobost@uned.ac.cr

Recibido: Enero 2014 • Aceptado: Mayo 2014

\section{RESUMEN}

Este artículo aborda un tema esencial: el respeto de los derechos de la niñez y de la adolescencia como una obligación por parte de la sociedad costarricense, ya que a diario estos derechos se ven violentados de distintas formas, tales como la explotación sexual comercial y la trata de personas. Dado que este es un asunto que concierne a toda la sociedad -incluyendo a las universidades como parte de la acción social que deben cumplir- la Escuela de Ciencias Sociales y Humanidades (ECSH) de la Universidad Estatal a Distancia (UNED) ha puesto en marcha desde el año 2010 un proyecto orientado a promover la reflexión y atacar desde la academia el irrespeto de los derechos de la niñez y de la adolescencia, y con ello tratar de fomentar la concientización y la realización de acciones dirigidas a prevenir distintas formas de violencia contra una de las poblaciones más vulnerables, mediante el trabajo con estudiantes, académicos e investigadores de distintas instituciones nacionales. Esto ha incrementado la cantidad de personas interesadas en la temática, con lo que se ha logrado promover el estudio y la reflexión al respecto.

Palabras clave: derechos, abuso, violencia, niñez, adolescencia, víctimas, intercátedras.

\section{ABSTRACT}

This article addresses a key issue: the respect for the rights of children and adolescents as an obligation by the Costa Rican society these are daily violated in different ways; one of the cruelest is the commercial sexual exploitation and trafficking. Since this is a matter that concerns all society -including universities as part of the social action they must meet-, the School of Social Sciences and Humanities at the Universidad Estatal a Distancia (UNED) has launched since 2010 a project to promote reflection and attack from the academy the disrespect for the rights of children and adolescents, and thus seek to promote awareness and implementation of measures aimed at preventing different forms of violence against one of the most vulnerable populations, 
any working with students, academics and researchers from different national institutions. It has resulted not only in the involvement of a large number of people in the field, but also has succeeded in promoting the study and reflection of this issue.

Key words: rights, abuse, violence, children, adolescents, victims, intercathedra.

\section{Introducción}

En el año 2010 se reúne un conjunto de cátedras pertenecientes a la Escuela de Ciencias Sociales y Humanidades (ECSH), con el propósito de configurar un proyecto que lleve a la reflexión y al análisis de diversos tipos de violencia, para crear formas de involucrar a los estudiantes de la Universidad Estatal a Distancia (UNED) en la lucha por la erradicación de dicha violencia y el cumplimiento de los derechos asociados a estas. El proyecto inicia con el nombre "Acerquemos a nuestros estudiantes a la problemática del abuso y la explotación sexual comercial".

El proceso inicia con la participación de los encargados de las cátedras de Psicología Educativa, Psicopedagogía, Ciencias Penales, Historia, Trabajo Social, Gestión Turística Sostenible y Formación Cívica y Geografía. En un primer momento se organiza el proyecto en torno a la búsqueda de información y documentación relevante sobre la problemática de la violencia, en especial de la explotación sexual comercial infantil y de la trata de personas, al considerarse mayormente invisibilizadas, pero posteriormente el proyecto se configura como una forma en que los estudiantes, mediante la asignación de proyectos de investigación y de trabajos de campo establecidos en las asignaturas de las cátedras, se involucraran en la temática y contribuyeran con sus aportes.

En el 2011 se continúa con el proceso anteriormente citado y se organiza una plenaria a la que asisten estudiantes universitarios y población académica de la UNED para conocer los proyectos realizados. Asimismo, se organiza una exposición itinerante de fotografías, como resultado del Proyecto de Prevención del Abuso y la Explotación Sexual Comercial Infantil en la Zona Sur de Costa Rica, específicamente en Ciudad Neily y Corredores.

En el 2012 se integran al trabajo las cátedras de Psicología, Artes, Métodos de Estudio a Distancia e Investigación, Secretariado, Derecho, Criminología, Teología y Comunicación Oral y Escrita, quienes llegan a colaborar con el crecimiento del Proyecto Intercátedras, dados los buenos resultados que hasta el momento se habían obtenido, con lo cual se logra mayor respaldo por parte de la autoridades de la Escuela y de la Universidad misma.

Para el año 2013 el proyecto continúa con su puesta en práctica, aportando la experiencia acumulada para fortalecerlo y posicionarlo en la ECSH; sin embargo, al finalizar el proceso se hizo necesario hacer una pausa para evaluar los resultados concretos y hacer los ajustes pertinentes, con la intención de retomarlo posteriormente (2015) con una mayor calidad.

\section{Marco referencial}

El Proyecto Intercátedras ha tomado como base la problemática de violencia, y se le ha dado prioridad a los temas de explotación sexual comercial infantil y a la trata de personas. Sin embargo, no por ello se excluyó el abuso en sus diferentes manifestaciones (física, sexual, emocional, psicológica, patrimonial o por negligencia), dependiendo de los intereses y necesidades propias de las cátedras participantes.

Con la intención de manejar un lenguaje común entre las cátedras, y con el fin de los estudiantes tuvieran uniformidad de conocimientos en las definiciones, se trató de sistematizar conceptos e indicadores asociados a la trata de personas y la explotación sexual comercial infantil que sirvieran como base para las propuestas de 
investigación y de trabajos de campo que debían realizar, esto siempre y cuando la cátedra considerara pertinente utilizar el material de acuerdo con sus intereses.

Se tiene claridad de que la definición de estos conceptos por sí mismos es insuficiente para su adecuado manejo, por ello es pertinente conocer los principales indicadores físicos y emocionales que contribuyen a la identificación de las problemáticas en las víctimas, así como a ciertos mitos del imaginario colectivo y a una breve caracterización de las personas que cometen abusos, de forma tal que se les ampliara a los estudiantes el panorama teórico.

A través de la información referencial entregada al estudiante, se pretende crear conciencia de que, cuanto más pronto se detecte la situación de abuso y violencia, más rápido este se podrá detener y atender al niño, a la niña o al adolescente para que supere el dolor causado por mantener durante un periodo de tiempo el terrible y doloroso secreto que ha llevado consigo.

La niñez es considerada como vulnerable ante situaciones de violencia por las relaciones asimétricas de poder en las que se encuentra, además de las desigualdades de género y de edad, lo cual le imposibilita buscar ayuda, situación de la que no están exentos los adolescentes, quienes son igualmente vulnerables.

Según la Fundación Paniamor (2000), las niñas y los niños más vulnerables de ser víctimas han nacido de forma prematura, son menores de cinco años, han sido víctimas de abuso anterior, han sufrido separación temprana y prolongada de su madre, presentan dificultades alimentarias, son adoptados, habitan en hogares sustitutos, se encuentran en condición de callejización, presentan necesidades educativas especiales, tienen problemas de salud, no manejan información y tienen baja autoestima.

A lo anterior, se ha de sumar la ausencia de redes de apoyo familiares, comunales y educativas que puedan ser soporte y apoyo en la prevención, factores que se acrecientan cuando de adolescentes se trata.

\section{Explotación sexual comercial infantil y trata de personas}

La explotación sexual comercial infantil se define como la

Utilización sexual de las personas menores de edad, donde medie un beneficio (o promesa de beneficio) económico o en especie, para la niña, niño, adolescente o persona intermediaria. Esta modalidad de comercio sexual puede manifestarse bajo distintas formas, tales como la venta y la trata, la pornografia, los espectáculos sexuales o la realización de actividades sexuales remuneradas. Los explotadores sexuales pueden ser residentes de la misma localidad donde viven las víctimas o desplazarse a otras zonas del país -turismo sexual-en su búsqueda. A la vez pueden abusar mediante el contacto directo con la víctima o bien, por medio del contacto indirecto a través de sus imágenes (pornografia) (OIT) IPEC, 2004: 5).

Es importante mencionar que hay varias formas de explotación sexual comercial: prostitución infantil, pornografía infantil, turismo sexual, tráfico de personas menores de edad y trata de personas (esta última se desarrolló como tema prioritario del Proyecto Intercátedras, por lo que se le hace mención aparte).

Este tipo de abuso, al igual que los anteriores, es una clara violación al derecho de las de víctimas a disfrutar su niñez y su adolescencia, y a llevar una vida libre de violencia. Además, la explotación sexual comercial podría acarrear consecuencias permanentes que amenazan la integridad personal y el desarrollo físico, emocional y social de las víctimas.

Dado lo anterior es que hay leyes que buscan proteger a los menores, tales como la Ley contra la explotación sexual de las personas menores de edad, la cual reformó varios artículos del Código Penal (156, 159, 160, 161, 162, 167, 168, $169,170,172,173$ y 174) con el fin de tipificar como delito las diversas manifestaciones de la explotación sexual comercial contra las personas menores de edad. Esto provee al país de un instrumento legal que permite perseguir, investigar, incriminar y penalizar acciones relacionadas con 
esta actividad que destruye la vida de niños, niñas y adolescentes.

En esta problemática se evidencian una serie de indicadores que se plantean a continuación:

\section{Indicadores físicos de la explotación sexual comercial infantil}

Estos incluyen el embarazo en niñas y adolescentes; niñas, niños y adolescentes con infecciones de transmisión sexual, VIH o sida, irritación, dolor o infecciones constantes en los órganos genitales, pérdida del apetito y problemas nutricionales y de alimentación; heridas, hematomas, golpes, insomnio, enfermedades psicosomáticas como alergias, úlceras gástricas, inflamación en el colon e hipertensión, entre otras.

Los indicadores físicos que se han presentado son generales, y según se menciona en el Manual sobre la lucha contra la trata de personas para profesionales de la justicia penal elaborado por la United Nations Office on Drugs and Crime (UNODC) pueden variar dependiendo de la tipología de trata de la que sean objeto las víctimas, ya que tales indicadores pueden variar entre víctimas de trata para fines de explotación sexual, de explotación laboral, de servicio doméstico, de mendicidad o para comisión de delitos menores. Por ello

El grupo de edad típico de las personas que se sospecha son víctimas en un lugar determinado depende de la naturaleza de la trata y la demanda en el lugar donde se produce la explotación. Con algunas excepciones, cuanta más edad tenga una persona, tanto menor será la probabilidad de que el caso implique trata. Este factor es especialmente destacable en los casos de explotación sexual. Por lo general, los traficantes no se dedicarán a la trata de personas de más edad con fines de explotación sexual porque existe poca "demanda de los clientes". Se han constatado algunas excepciones en las que el mercado "cliente" considera que personas mayores de una etnia concreta tienen un aspecto juvenil. La misma regla general puede aplicarse a la explotación laboral, porque cuanta más edad tenga la persona, tanto menos productiva será en condiciones de trabajo arduo o de esclavitud. Sin embargo, hay excepciones: la trata de personas mayores con fines de mendicidad es un ejemplo. Los niños son particularmente vulnerables a la trata, porque pueden ser dóciles y se les puede explotar de varias maneras: en la industria del sexo, en los mercados laborales ilegales, con inclusión de la mendicidad y del carterismo; como "esclavos" domésticos y para extraerles órganos (UNODC, 2010: 5 ).

\section{Indicadores emocionales de la explotación sexual comercial infantil}

Dentro de los indicadores emocionales se pueden anotar aspectos tales como depresión, ansiedad y agresividad; uso de ropa muy llamativa y mucho maquillaje para salir, en el caso de las adolescentes; bajo rendimiento, deserción escolar y ausencias injustificadas al centro educativo; huidas -escapar- de la casa, salidas por las noches en compañía de personas adultas con un regreso tarde; realizar o recibir llamadas frecuentes de hombres desconocidos -nacionales o extranjeros-; dificultades de concentración, baja autoestima, gran interés por temas sexuales; ingesta de licor o uso de drogas; exhibición de objetos, ropa o dinero que van más allá de las posibilidades económicas que posee la familia.

Por su parte, la trata de personas se define de la siguiente forma:

Por trata de personas se entenderá la captación, el transporte, el traslado, la acogida o la recepción de personas, recurriendo a la amenaza o al uso de la fuerza u otras formas de coacción, al rapto, al fraude, al engaño, al abuso de poder o de una situación de vulnerabilidad o a la concesión o recepción de pagos o beneficios para obtener el consentimiento de una persona que tenga autoridad sobre otra, con $\mathrm{fi}_{\mathrm{i}}$ nes de explotación. Esa explotación incluirá, como mínimo, la explotación de la prostitución ajena $u$ otras formas de explotación sexual, los trabajos o servicios forzados, la esclavitud o las prácticas análogas a la esclavitud, la servidumbre o la extracción de órganos (ONU, 2004, art. 3).

Así, la trata de personas se refiere a toda persona, de cualquier sexo, edad y procedencia que realiza, individual o grupalmente $-\mathrm{O}$ como 
parte de una red nacional o internacional y con pleno conocimiento de las consecuencias de sus actos- las siguientes conductas típicas: captación o reclutamiento de personas para ser tratadas, traslado, transporte, retención o recepción de víctimas.

Es necesario tener en consideración que puede ser víctima de trata de personas cualquier ser humano, sin distinción de sexo, edad, nacionalidad, lugar de procedencia, entre otras características.

En la trata de personas también se evidencian algunos indicadores que se enuncian a continuación:

\section{Indicadores físicos de la trata de personas}

Entre estos indicadores se puede observar que las víctimas también lo son de otros tipos de violencia, como física y emocional, cuyos indicadores son similares a los anteriormente mencionados: desnutrición, pérdida del deseo de ingerir alimentos, ojeras, cansancio, desmejoramiento físico, problemas de salud física y mental, fatiga, pérdida de peso, síntomas neurológicos y problemas gastrointestinales.

\section{Indicadores emocionales de la trata de personas}

Los indicadores de este tipo incluyen manifestaciones tales como que la persona cree que no puede salir de la situación, da muestras de angustia, temor, ansiedad y desconfianza, se siente amenazada, o cree que va a ser amenazada o que su familia está siendo amenazada o va a ser amenazada si se revela. La persona presenta lesiones que parecen causadas por agresiones, no puede trasladarse de lugar de residencia, está aislada de todo sistema de apoyo, carece de lo indispensable, como alimentos, agua, refugio y atención médica; está endeudada, sin posibilidad de pagar la deuda. Además, la persona no puede conseguir documentos de identificación como el carné de identidad, el permiso de conducir o el pasaporte (UNODC, 2010).

\section{Mitos asociados con la violencia y el abuso}

Cuando se trabaja con situaciones de violencia, es frecuente enfrentarse con "verdades falsas" entre las personas que son víctimas de estos flagelos o en sus familias, que contribuyen a que las situaciones se sigan manteniendo en secreto e imposibilitan que se brinde una atención adecuada y pronta.

Se les puede llamar "verdades falsas", porque las personas creen que las frases dichas para justificar las acciones de la persona agresora, o las expresadas por la familia, por la comunidad y por la sociedad son ciertas, por lo que estas falsedades dañan a la víctima, le acrecientan sus inseguridades, miedos y temores, minan su autoestima e inclusive, como se mencionó anteriormente, las hace mantener por más tiempo el secreto.

Un mito es una percepción falsa de la realidad que se acepta como verdadera (Garaigordobil, 2010), es así que en el caso de la violencia y del abuso, la persona agresora elabora un relato genuino y totalmente creíble acerca de la importancia de no revelarle a nadie el secreto que comparten, lo cual se acompaña de falsas promesas o amenazas.

El agresor se siente muy seguro de sí mismo y en apariencia su autoestima es alta, pero esta seguridad surge de la relación desigual y asimétrica de poder que tiene con la víctima, lo cual le genera esa confianza adicional para sortear los obstáculos que se presenten y calcular los momentos para abusar de su víctima, a sabiendas de que esta no revelará su realidad. Conoce bien a la víctima, la ha analizado y sabe cuáles son sus puntos débiles e hilos más delgados, con los cuales la puede manipular para seguir realizando sus actos.

En ocasiones, incluso, la persona abusadora goza de estima y reconocimiento dentro del círculo familiar, sea o no integrante de este, ya que se ha ganado el respeto y cariño de las personas a través de obsequios, presentes, aportes económicos y otras manifestaciones que le han hecho verse como alguien importante y preocupado por su bienestar.

Como ya se mencionó, suele hacerse referencia a las "verdades falsas" como mitos o 
estereotipos, siendo estos últimos definidos como la creencia acerca de la imagen que se tiene de algo y que se toma como verdad (Garaigordobil, 2010), es decir, como aquellas frases que escuchamos en las voces populares y que repetimos como verdades absolutas sin cuestionar ni reflexionar acerca de su veracidad.

En la experiencia profesional y a través de la lectura de información relacionada con la temática, se han encontrado algunos de los mitos

\section{CUADRO 1}

Mitos, estereotipos y verdades asociados al abuso

Mitos-estereotipos
Los niños y las niñas provocan que la
persona adulta o mayor abuse de ellos.
Los niños y las niñas fantasean y
mienten acerca del abuso que les está
sucediendo.
La persona abusadora consume drogas
o alcohol.

Las personas abusadoras son solo de nacionalidad extranjera.

Las personas solo maltratan a los niños y a las niñas cuando han tenido un día particularmente difícil o estresante.

A las niñas les gusta tener sexo con la persona abusadora.

Cuando sea más grande, se acabará y dejará de abusarme.

Solo las niñas son abusadas sexualmente.

Las personas agreden porque en su niñez también fueron agredidas.

Si el abuso sucede en el interior de la familia, se sabrá más pronto lo que pasa.

\section{Verdades}

Se debe respetar a las personas sin importar la edad que se tenga. Es respeto del cuerpo, de la integridad de la niñez.

La niñez no suele mentir acerca de las situaciones de abuso que le suceden, en especial cuando se trata de abuso sexual.

Las personas abusadoras son seres humanos que con premeditación cometen los actos de violencia y abuso, creyendo que la relación de poder desigual que tienen las va a librar de la revelación de secretos por parte de la víctima. Normalmente calculan cuándo van a cometer el abuso y de qué forma.

Las personas abusadoras son cualquier persona, sin importar sexo, etnia, nacionalidad o credo religioso.

Las personas ofensoras son agresoras. Las personas somos quienes justificamos las acciones que estos cometen.

Los niños y las niñas sienten temor de denunciar los hechos porque piensan que no les creerán. Existe dependencia emocional y económica con estas figuras. La relación de poder desigual existe.

El tiempo que transcurre desde la primera vez que la víctima es abusada y la ruptura del secreto es en promedio de 2 a 3 años, por lo que el tiempo del abuso se prolonga. Hay situaciones en que los daños psicológicos de las víctimas son tan severos, porque los abusos han continuado hasta edades de adolescencia e incluso adultez.

Si bien es cierto las niñas son quienes sufren más este tipo de abuso, en los niños es cada vez más frecuente. Lamentablemente, a los varones -por socialización de género- les es más difícil hacer la revelación.

Si esto fuera cierto, las mujeres serían quienes más agreden a la niñez, ya que -por porcentajes-son las más vulnerables y quienes más lo han sufrido. Los estudios citan que son los hombres quienes más agreden.

No siempre sucede de esa forma, ya que el abuso se da bajo el mandato del secreto y con amenazas para que no se dé su ruptura. Además, conoce la dinámica familiar y cómo actúan las personas, lo que le hace calcular los tiempos para cometer los actos. 
CUADRO 1 (Continuación...)

Mitos, estereotipos y verdades asociados al abuso

\section{Mitos-estereotipos}

Las agresiones se dan en familias pobres y analfabetas.

La persona agresora es una desconocido.

Los efectos en las víctimas se superan fácil y rápidamente.

Sucede pocas veces.

El abuso es una situación privada que debe mantenerse en el interior de la familia.

\section{Verdades}

El abuso y la violencia ocurren en todos los estratos sociales. La diferencia radica en que, en clases más altas, se mantiene en secreto y se buscan apoyos privados. Por otra parte, la influencia cultural existe, por lo que en zonas urbanas se habla más de estos temas, mientras que en las rurales se sigue considerando un tabú.

No necesariamente es así, ya que en ocasiones son los padres, madres, padrastros, tíos, abuelos; es decir, personas del círculo familiar o allegados a este.

Los efectos varían en cada persona, y dependen de factores como la edad, tipo de abuso, forma en que este se cometió, reacción de la familia, tiempo de revelación, redes de apoyo, atención psicológica recibida, entre otras. Se ha de destacar que las secuelas existen y son permanentes, siendo lo verdaderamente importante el que la víctima reciba apoyo profesional adecuado de forma pronta.

Los abusos se dan de manera reiterada si las condiciones que suscitaron los hechos se mantienen de igual forma. Si hay cambios y rupturas en las dinámicas puede ser que los abusos no se repitan.

La denuncia es un paso importante para la recuperación de la víctima, además de ser una forma para que se apoye a la familia y a la víctima para solventar las problemáticas.

Fuente: Elaboración propia.

y estereotipos asociados con el abuso que más se repiten. Estos se muestran en el cuadro 1 (se presenta asimismo su desmitificación).

\section{Personas que cometen diversos tipos de abuso}

Quienes agreden y lastiman a niños y a niñas pueden ser cualquier persona. Sin embargo, los que se encuentran más cerca de los menores son quienes suelen cometer en mayor número dichos maltratos.

El abuso se puede dar tanto en la familia como en otros ámbitos en los que se desarrolle la niñez o adolescencia, por lo que se debe ser vigilante de las conductas e indicadores, ya que son evidentes los cambios físicos y de comportamiento que pueden presentar tanto los menores de edad como sus padres o personas encargadas que resulten sospechosas de ser abusadores, lo cual sin duda debe ser corroborado.

\section{Motivos que generan conductas de abuso}

Según varias fuentes, entre ellas FAI-SavetheChildren (2007), los abusos pueden ser perpetrados, entre otros, por los siguientes dos aspectos:

1. Congruencia emocional: las personas abusadoras, por su inmadurez emocional, encuentran satisfacciones emocionales en los niños y en las niñas. Estas necesidades se originan en la baja estima y el deseo de control, entre otros aspectos.

2. Bloqueo: las personas agresoras orientan sus deseos sexuales hacia niñas y niños en la 
medida en que se sientan imposibilitadas de mantener relaciones sexuales con adultos.

Por lo tanto, los abusos cometidos se basan en el poder y control que la persona adulta tiene sobre sus víctimas, al considerarles seres inferiores y fácilmente manipulables.

\section{Sistematización de la experiencia desde la Escuela de Ciencias Sociales y Humanidades (ESCH) de la UNED}

Como se explicó en la introducción, el Proyecto Intercátedras comenzó en el año 2010 como iniciativa de seis cátedras de la Escuela de Ciencias Sociales y Humanidades. Del proceso organizativo iniciado se derivó la necesidad de formular objetivos por lo que se plantearon los siguientes:

\section{Objetivo general}

Difundir aspectos fundamentales sobre abuso, explotación sexual comercial infantil y trata de personas menores de edad como formas de violación a los derechos de niños, niñas y adolescentes, con el fin de crear conciencia en la población estudiantil de las asignaturas que brinda la Escuela de Ciencias Sociales y Humanidades y en la población en general.

\section{Objetivos específicos}

- Analizar las problemáticas de abuso, explotación sexual comercial infantil y trata de personas menores de edad como formas de violación a los derechos de niños, niñas y adolescentes, desde una perspectiva que permita trascender el enfoque patriarcal de la sociedad, mediante la incursión de actividades de investigación en las asignaturas de la Escuela de Ciencias Sociales y Humanidades.

- Difundir los conocimientos teóricos, las manifestaciones y las experiencias de investigación por parte de docentes y estudiantes sobre los temas del Proyecto Intercátedras, mediante recursos audiovisuales y virtuales.
- Generar conciencia en el personal docente y administrativo sobre la realidad de la violación a los derechos humanos que padece la población menor de edad.

- Brindar los elementos fundamentales que permitan al estudiantado involucrarse en la lucha contra estas formas modernas de esclavitud. (Comisión Intercátedras, 2012)

A partir de la formulación de los objetivos anteriormente planteados, se inició en el 2011 la ejecución mediante la asignación de proyectos de investigación y trabajos de campo a los estudiantes de diferentes asignaturas de las seis cátedras. Luego, se eligieron las mejores propuestas para ser presentadas en la plenaria organizada para este efecto. Esta actividad fue acompañada por la exposición itinerante de fotografías resultado del Proyecto Prevención del Abuso y la Explotación Sexual Comercial en la Zona Sur de Costa Rica, específicamente en Ciudad Neily y Corredores.

En el año 2012 con la incorporación de más cátedras y después de la revisión, discusión y análisis de los objetivos del proyecto se plantea nombrarlo Es Asunto de Todos y de Todas. Las acciones concretas planteadas para ese año se circunscribieron a aspectos como:

1. Esbozo de un marco teórico que brinde sustento al proyecto.

2. Planteamiento de investigaciones y trabajos de campo desde distintas asignaturas para ser aplicadas en diversos medios como escuelas, colegios, comunidades, lugares de trabajo. Las investigaciones se plantearon desde diversas disciplinas y al finalizar se eligieron los trabajos que alcanzaron un mejor acercamiento al tema. Se invitó a los estudiantes a presentar su investigación en la celebración institucional del Día Mundial contra la Explotación Sexual Comercial.

3. Contacto con otras instancias como el Patronato Nacional de la Infancia (PANI), la Comisión Nacional Contra la Explotación Sexual Comercial (CONACOES), el Ministerio de Relaciones Exteriores, específicamente 
con el área de Derechos Humanos y Seguridad Humana de la Dirección General de Política Exterior.

4. Elaboración y alimentación de un de un blog para la divulgación en red del proyecto y de la temática en sí.

5. Elaboración de programas de radio y videoconferencias.

6. Divulgación del proyecto a instituciones educativas de III y IV ciclo de la Enseñanza General Básica.

7. Invitación de expertos en el tema, con el fin de que se difundiera conocimiento con suficiente sustento académico acerca de la temática.

8. Actividades de cierre anual para la divulgación de las investigaciones realizadas y la sensibilización de la comunidad universitaria y otras poblaciones invitadas.

Así mismo, se da la autoevaluación del trabajo realizado, cuyo resultado principal fue la importancia de continuar trabajando los temas de la explotación sexual comercial infantil y la trata de personas, pero acompañado de otras temáticas que revisten de importancia para el momento coyuntural por el que atraviesa la sociedad y el país en que se presenta una crisis innegable en distintos ámbitos como lo social, lo político, lo económico y el de los valores, entre otros.

Acompañado de este proceso de autoevaluación, las cátedras participantes reciben una conferencia por parte de la doctora Ana Teresa León, docente y terapeuta de niños, investigadora sobre los derechos integrales de las personas menores de edad; además cuenta con una trayectoria de muchos años en temas de niñez y adolescencia y recientemente ha sido designada como presidenta ejecutiva del Patronato Nacional de la Infancia. Fue invitada por la Comisión de Extensión de la ESCH para que expusiera sobre el tema a las personas encargadas de cátedra involucradas en el Proyecto Intercátedras. La doctora León concordó con la necesidad expuesta en el párrafo anterior, respecto a la necesidad de evidenciar otras dificultades que enfrentan la niñez y la adolescencia, tales como abandono, exclusión educativa, violencia intrafamiliar, deficientes controles de salud, embarazo adolescente, sistemas educativos excluyentes, explotación laboral, condiciones de vida infrahumanas, drogadicción y pocas oportunidades de ascenso social.

Así, es como se plantea que el Proyecto Intercátedras Es Asunto de Todos y de Todas ampliara sus horizontes de investigación, reflexión y divulgación hacia los derechos de la niñez y de la adolescencia, y no se limitara tan solo al ámbito de la explotación sexual comercial infantil y la trata de personas (aunque por supuesto esta sigue siendo una temática de interés dentro del trabajo que se realiza). A la vez se revisan los objetivos del proyecto y se mantienen al ser congruentes con esta renovada perspectiva.

Para el año 2013 se continúa con las estrategias de los años anteriores pero se amplía a actividades como:

1. Concurso de "memes" en el perfil de Facebook del Patronato Nacional de la Infancia, a partir del tema de derechos de la niñez y de la adolescencia.

2. Fortalecimiento del blog Es Asunto de Todos y de Todas, el cual es alimentado con información relevante sobre las temáticas del proyecto por parte de las cátedras participantes.

3. Búsqueda de alianzas con otras instancias, tales como el Hospital Nacional de Niños, el PANI y la Revista Espiga de la ECSH, para el tratamiento y divulgación del proyecto y de las temáticas tratadas.

4. Dada la relevancia del tema se gestiona y se hace posible el reconocimiento del proyecto como de interés institucional por parte del Consejo de Rectoría de la Universidad.

Al finalizar el proceso de ese año, se hizo necesario hacer una pausa para nuevamente evaluar los resultados y de esta forma realizar los ajustes pertinentes para retomarlo posteriormente (2015), con las mejoras que del proceso evaluativo y reflexivo se deriven. 


\section{Productos obtenidos con el Proyecto Intercátedras}

A lo largo de los cuatro años de ejecución del proyecto, se alcanzaron productos importantes, siendo estos los siguientes:

1. La declaración del proyecto como de interés institucional de modo permanente; lo cual le aporta un rango de importancia y de reconocimiento a la labor efectuada, así como la posibilidad de mayor difusión a partir del acuerdo tomado por el Consejo de Rectoría en sesión n. ${ }^{\circ}$ 1774-2013 (artículo III, inciso 11), celebrada el 10 de junio de 2013 (CONRE, 2013a).

2. Participación de diversas cátedras desde las que se han planteado investigaciones y trabajos de campo por parte de los estudiantes, referente a los temas de explotación sexual comercial infantil, trata de personas y derechos de la niñez y de la adolescencia.

3. Elaboración y difusión de programas de radio.

4. Elaboración y difusión de videoconferencias.

5. Creación y alimentación del blog Es Asunto de Todos y de Todas, con difusión en la web. Dicho blog contiene variedad de temas de relevancia acerca de la explotación sexual comercial infantil y la trata de personas, pero también sobre otros temas relacionados con los derechos de la niñez y de la adolescencia. El sitio presenta un promedio mensual de 325 visitas, provenientes de Costa Rica, México, Colombia, EEUU, Argentina, Perú, El Salvador, Chile, España, Ecuador, Venezuela, Nicaragua, Paraguay, Panamá, Puerto Rico, Bolivia y Guatemala. ${ }^{1}$

6. Divulgación de la temática a estudiantes y docentes de escuela y colegio y a comunidades, ello mediante las investigaciones y trabajos de campo realizados por los alumnos

1. Datos suministrados por Pablo Bonilla, encargado de la Cátedra de Artes y administrador del blog. de la UNED en esos contextos, así como la invitación de poblaciones de secundaria a las actividades de cierre realizadas en los años 2012 y 2013.

7. Actividades de cierre anuales para divulgar y reflexionar sobre las temáticas que sustentan al proyecto. Se han dado tres actos de esta naturaleza: la primera en noviembre del 2011, donde se contó con la participación de siete estudiantes de diversas cátedras que expusieron sus investigaciones referentes al tema de la explotación sexual comercial infantil y la trata de personas; la segunda en setiembre del 2012, en el marco de la conmemoración del 23 de setiembre, Día Contra la Explotación Sexual Comercial. Para esta jornada se organizó un panel que contó con la participación de tres expertas cuyas exposiciones abordaron los siguientes temas:

a) La realidad de la explotación sexual comercial en niños, niñas y adolescentes en Costa Rica, a través de un análisis desde los derechos humanos de las personas sometidas a esas condiciones de violencia.

b) El impacto sicológico y el proceso de recuperación para las víctimas.

c) La situación nacional, cómo se trabaja el tema, cuál es el impacto social, cuáles son las acciones de prevención, etcétera.

Las expositoras fueron Eugenia Gutiérrez, encargada de Derechos Humanos y Seguridad Humana de la Dirección General de Política Exterior del Ministerio de Relaciones Exteriores y Culto; Nora González, encargada del programa Agenda Joven de la UNED; y Elizabeth Ballestero, de CONACOES.

8. Actividad en conmemoración de la Declaración de los Derechos del Niño (1959) y la Convención sobre los Derechos del Niño (1989), la cual fue realizada en noviembre de 2013, con Ana Virginia Quesada Morales, tutora de la cátedra de Trabajo Social y directora del 
Departamento de Trabajo Social del Hospital Nacional de Niños, como expositora.

9. Además, reserva del número 28 de la Revista Espiga para la publicación de artículos tanto de estudiantes unedianos cuyos trabajos fueron realizados en el marco del Proyecto Intecátedras, como de investigaciones y disertaciones temáticas de académicos y académicas de distintas instancias de la UNED y de otras instituciones.

10. Se coordina una alianza estratégica con diferentes instituciones tanto para la exposición de especialistas en el marco del proyecto como para el acercamiento a las poblaciones meta. Lo anterior se evidencia mediante el concurso de "memes" planteado desde el perfil de Facebook del PANI, cuya premiación se realizó en noviembre del 2013.

11. El fortalecimiento del proyecto con la participación real y activa de las cátedras de Psicología, Psicopedagogía, Psicología Educativa, Trabajo Social, Derecho, Ciencias Penales, Secretariado Administrativo, Formación Cívica y Geografía, Historia, Criminología, Enseñanza del Inglés, Gramática y Literatura.

12. Por otro lado, siempre en el tema de la niñez y la adolescencia se planteó, de parte de la encargada de Extensión de la ECSH, a la comisión coordinadora del proyecto, la colaboración en la I Carrera por los Derechos de la Niñez y la Adolescencia Costarricense, cuyo proyecto se acordó por parte del Consejo Universitario en la sesión n. ${ }^{\circ}$ 1784-2013 (CONRE, 2013b).

\section{Conclusiones}

1. Dado que los derechos de la niñez y la adolescencia no son una opción, sino un tema de acatamiento obligatorio por parte de las personas adultas, la sociedad costarricense en conjunto debe velar por su cumplimiento. Es por ello que las universidades deben asumir un papel protagónico en la lucha por los derechos de las personas, especialmente de las más vulnerables. Una forma de asumir ese papel es promover la reflexión entre los futuros profesionales, así como el acercamiento a distintos contextos para -desde la academia- promover el análisis y la prevención de la violencia contra la niñez y contra la adolescencia, tal y como se ha promovido la reflexión de esta temática desde el Proyecto Intercátedras de la ECSH.

2. La prevención es un factor determinante que no se debe obviar: cuanto más se hable acerca de estas temáticas se estará contribuyendo al empoderamiento de la niñez, para evitar que sea vulnerable. Estos temas son igualmente importantes para que sean abordados con las personas responsables de los menores de edad, para que les faciliten contactos, lugares y personas de referencia que podrían apoyar en estas situaciones y que se encuentran cercanos a su comunidad.

3. La prevención y atención de la violencia y el abuso es responsabilidad de todas las personas, pero aún más de quienes trabajan directamente con la niñez. Así, proyectos como Es Asunto de Todos y de Todas logran despertar el interés de la academia, la alianza de distintas instancias e instituciones y la promoción de la reflexión de próximos profesionales en pro de los derechos de las personas menores de edad, no solo en temáticas como la explotación sexual comercial infantil y la trata de personas, sino de otras falencias y agresiones que sufren estas poblaciones.

\section{Bibliografía}

Claramunt, M. (1998). Explotación sexual en Costa Rica. Análisis de la ruta crítica de niños, niñas y adolescentes hacia la prostitución. Costa Rica: UNICEF.

Comisión InTERCÁtedras (2012). Documento del proyecto Intercátedras. ECSH. UNED. sp.

Fai-Savethechildren (2007). "Prevención de abuso sexual en niños y niñas. Una perspectiva con enfoque de derechos". Recuperado de <http://www.slideshare.net/ demysex/abuso-sexual-de-nios-y-nias $>$. Consultado el 22 de agosto de 2013. 
Fundación PANiAmor (2000). Guía para la prevención del abuso infantil desde la escuela. San José.

Garaigordobil, M. (2010). "Un estudio correlacional de las cogniciones prejuiciosas con diversas conductas sociales y con rasgos de personalidad". Anuario de Psicología, vol. 31, n. ${ }^{\circ}$ 3: 39-57.

OIT / IPEC (2004). Documento básico de información sobre la problemática de la explotación sexual comercial de niños, niñas y adolescentes: Centroamérica, Panamá y República Dominicana. OIT/IPEC. San José.

ONU (2004). Protocolo para prevenir, reprimir y sancionar la trata de personas, especialmente mujeres y niños, que complementa la Convención de las Naciones Unidas contra la delincuencia organizada transnacional (Protocolos de
Palermo). Recuperado de http://www.unodc.org/documents/treaties/UNTOC/Publications/TOC\%20Convention/TOCebook-s.pdf>. Consultado el 22 de agosto, 2013

Uned (2013a). Consejo de Rectoría, acuerdo n. ${ }^{\circ}$ 425-2013, 10 de junio.

Uned (2013b). Consejo de Rectoría, acuerdo n. ${ }^{\circ}$ 693-2013, 26 de agosto.

UnodC (2010). Manual sobre la lucha contra la trata de personas para profesionales de la justicia penal. Módulo 2. Nueva York. Recuperado de <http://sicarcat.wordpress. com/2012/10/29/manual-sobre-la-lucha-contra-la-tratade-personas-para-profesionales-de-la-justicia-penal/> . Consultado el 13 de setiembre de 2013. 